\title{
Factores pronósticos de complicaciones en pacientes con cardiopatía isquémica sometidos a anestesia total intravenosa en cirugía electiva
}

\section{Total intravenous anethesia in ischemic coronary patients: Prognosis factors}

\author{
José Miguel Torres Anaya1, Israel Tarancón Serrano², Yacnira Loreleis Martínez Bazán ${ }^{3, *}$, C. Yurisnel Ortiz Sánchez ${ }^{4}$ \\ Especialista en primer grado en Anestesiología y Reanimación y en Medicina General Integral. \\ 2 Especialista en primer grado en Anestesiología y Reanimación. Profesor Asistente. \\ 3 Especialista en 2do grado en Anestesiología y Reanimación. Profesor e investigador Auxiliar. Master en Urgencias Médicas y en \\ Acupuntura. \\ 4 Profesor Titular. \\ Hospital provincial Carlos Manuel de Céspedes de Bayamo, Cuba.
}

Fecha de recepción: 12 de agosto de 2021 / Fecha de aceptación: 21 de octubre de 2021

\begin{abstract}
A prospective longitudinal cohort study was conducted in patients with ischemic heart disease undergoing total intravenous anesthesia in elective surgery at the "Carlos Manuel de Céspedes Bayamo Provincial Hospital, from the Cauto region during the period from January 1, 2015 to March 30, 2017; with the aim of identifying the risk factors hypothetically related to the prognosis of the appearance of anesthetic complications. The exposed cohort consisted of 47 patients who developed complications in the study period and met the inclusion criteria. To assess the association between the variables, the Mantel Chi-square test was used. The magnitude of the associations was estimated by calculating the relative risks (RR) of complications. The consumption of tobacco as a toxic habit, the non-use of beta-blockers and statins were the surgical risk factors depending on the patient associated with the prognosis of the appearance of anesthetic complications; not so age. Comorbidity in patients with ischemic heart disease of diabetes mellitus, heart failure and arrhythmias, were associated with the appearance of anesthetic complications. The ASA III-IV classification and high-risk surgical procedures were the surgical risk factors based on the surgery related to the prognosis of anesthetic complications.
\end{abstract}

Key words: Ischemic heart disease, hemodynamic complications, total intravenous anesthesia, elective surgery.

\begin{abstract}
RESUMEN
Se realizó un estudio longitudinal prospectivo de cohorte en pacientes con cardiopatía isquémica sometidos anestesia total intravenosa en cirugía electiva en el Hospital provincial "Carlos Manuel de Céspedes de Bayamo, procedentes de la región del Cauto durante el período comprendido desde el $1^{\text {ro }}$ de enero del 2015 hasta 30 de marzo de 2017. El objetivo era identificar los factores de riesgo hipotéticamente relacionados con el pronóstico de aparición de complicaciones anestésicas. La cohorte expuesta estuvo constituida por 47 pacientes que desarrollaron complicaciones en el período de estudio y cumplieron con los criterios de inclusión. Para valorar la asociación entre las variables, se empleó el test de Ji al Cuadrado de Mantel. La magnitud de las asociaciones se estimó mediante el cálculo de los riesgos relativos (RR) de complicaciones. El consumo de tabaco como hábito tóxico, el no uso de beta-bloqueadores y estatinas se constituyeron en los factores de riesgo quirúrgico en función del enfermo, asociados con el pronóstico de aparición de complicaciones anestésicas; no así la edad. La comorbilidad en los pacientes con cardiopatía isquémica de diabetes mellitus, insuficiencia cardíaca y las arritmias, se asociaron a la aparición de complicaciones anestésicas. La clasificación ASA III-IV y los procedimientos quirúrgicos de alto riesgo fueron los factores de riesgo quirúrgico en función de la cirugía relacionados con el pronóstico de aparición de complicaciones anestésicas.
\end{abstract}

Palabras clave: Cardiopatía isquémica, complicaciones hemodinámicas, anestesia total intravenosa, cirugía electiva. 


\section{Introducción}

$E^{n}$ n la práctica clínica diaria, con frecuencia se presentan casos en los que los profesionales de la salud, en el campo clínico (médicos internistas y familiares, cardiólogos, anestesiólogos y cirujanos), se ven involucrados en la valoración preoperatoria de pacientes complejos que poseen cierto riesgo de complicaciones cardiovasculares. Por tanto, es necesario estar preparados para la realización de una evaluación que reduzca la morbimortalidad pre-, peri- y pos-procedimiento, que fomente, además, el uso racional de recursos diagnósticos y terapéuticos, y que permita optimizar el tratamiento médico del individuo[1],[2].

La anestesia intravenosa total (TIVA o ATIV) es una técnica que permite diversidad de combinaciones de anestésicos según necesidades individuales de cada paciente, lo que constituye una herramienta más para disminuir la morbilidad perioperatoria en pacientes con factores de riesgo coronario[3],[8].

Considerando que la evaluación del riesgo quirúrgico es decisiva en la población con cardiopatía isquémica, y teniendo en cuenta que no se disponen de estudios en la provincia, se decide realizar la presente investigación para responder al siguiente problema científico: ¿Cuáles son los factores de riesgo hipotéticamente relacionados con el pronóstico de aparición de complicaciones anestésicas en pacientes con cardiopatía isquémica sometidos anestesia total intravenosa en cirugía electiva?

Se parte de la hipótesis de que: la edad, los hábitos tóxicos como el consumo de tabaco, enfermedades asociadas (diabetes mellitus, insuficiencia cardíaca, arritmias), el no uso de beta-bloqueadores y estatinas, la clasificación ASA y los procedimientos quirúrgicos de alto riesgo constituyen factores de riesgo relacionados con el pronóstico de aparición de complicaciones anestésicas en pacientes con cardiopatía isquémica sometidos anestesia total intravenosa en cirugía electiva.

\section{Diseño metodológico}

\section{Características generales de la investigación}

Se realizó un estudio longitudinal prospectivo de cohorte en pacientes con cardiopatía isquémica sometidos a anestesia total intravenosa en cirugía electiva en el Hospital provincial "Carlos Manuel de Céspedes de Bayamo, durante el período comprendido desde el $1^{\text {ro }}$ de enero de 2015 hasta 30 de marzo de 2017.

El universo estuvo formado por los 222 pacientes intervenidos por cirugía electiva con anestesia total intravenosa en el servicio de cirugía del Hospital Universitario "Carlos Manuel de Céspedes" de Bayamo, Granma, Cuba en el período antes mencionado.

Para el cálculo del tamaño de la muestra se diseñó un estudio de cohorte prospectivo.

Partiendo del universo de estudio se tomó una muestra probabilística y la selección de la cohorte se realizó mediante muestreo aleatorio simple.

Estableciendo una prevalencia del factor de exposición en el grupo no enfermo del 33\% y en el grupo enfermo del $23 \%$ con $95 \%$ de coeficiente de confianza que es igual a 0,05\% de nivel de significación del error, con una potencia de 80(1- $\beta$ ).

Se estableció que la cohorte expuesta estuvo constituida por 47 pacientes que desarrollaron complicaciones en el perío- do de estudio y cumplieron con los criterios de inclusión.

Para el cálculo del tamaño de la muestra se utilizó el paquete estadístico Epilnfo versión 2002 para Windows.

Selección de la cohorte expuesta: Se incluyeron consecutivamente todos los pacientes con diagnóstico de cardiopatía isquémica, intervenidos por cirugía electiva con anestesia total intravenosa, que expresen su consentimiento de participar en la investigación en el salón de cirugía general y que presentaron hallazgos de complicaciones anestésicas como consecuencia del acto quirúrgico.

La evaluación del diagnóstico de complicaciones se realizó durante todo el procedimiento quirúrgico y terminado este, y por lo general por dos especialistas de Anestesiología y Reanimación al unísono. Uno de ellos fue el que intervino inicialmente al enfermo cuando se realizó la evaluación inicial. De esta forma se previno el sesgo de evaluación en el estudio.

La recogida del dato primario se obtuvo de las historias clínicas de los pacientes intervenidos en el salón de cirugía general del hospital que participa en la investigación, se realizó por dos especialistas por separado y luego se confrontaron los datos. Los mismos se plasmaron en un formulario creado al efecto, que incluyó las variables seleccionadas. Estas variables fueron vaciadas posteriormente en una base de datos creada en SPSS versión 15.

\section{Resultados}

Se incluyeron 222 pacientes intervenidos por cirugía electiva con el empleo de anestesia total intravenosa y diagnóstico de cardiopatía isquémica en el salón de cirugía general del Hospital provincial "Carlos Manuel de Céspedes de Bayamo, procedentes de la región del Cauto durante el período de estudio, en los que 47 desarrollaron complicaciones anestésicas.

En la Tabla 1, se explica el resultado del análisis estadístico univariado realizado para identificar la posible asociación entre los factores de riesgo en función del enfermo con el pronóstico de aparición de complicaciones anestésicas, cuando dichas variables hipotéticas se manifiestan aisladamente en la población estudiada.

En relación a la edad por encima de 55 años, aunque obtuvo un valor del RR mayor de 1 (RR 1,56; IC 95\%: 0,84-2,88; $p=$ $0,1965)$, el resultado no fue estadísticamente significativo, por lo que no constituyó en la investigación en un factor pronóstico.

El tabaquismo se constituyó en un factor pronóstico de aparición de complicaciones anestésicas, al obtenerse un valor del RR significativamente mayor de 1 (RR 3,28; IC 95\%: 1,84-5,85; $\mathrm{p}=0,0000$ ), de modo que existió tres veces mayor probabilidad de desarrollar complicaciones anestésicas en los pacientes que consumen tabaco o sus derivados, que aquellos que no lo hacen.

La no prescripción de $\beta$-bloqueadores al menos de 2 a 7 días antes el procedimiento incrementa significativamente el riesgo de que aparezca complicaciones anestésicas (RR 2,40; IC 95\%: 1,32-4,86; $p=0,0045)$, constituyéndose en un factor pronóstico. El no consumo de estatinas, se constituye en un factor pronóstico al duplicar el riesgo de complicaciones anestésicas (RR 2,26; IC 95\%: 1,26-4,05; p = 0,0074).

La influencia de los factores de riesgo quirúrgico dependien- 
Tabla 1. Factores de riesgo quirúrgico en función del enfermo. Hospital Carlos Manuel de Céspedes. Análisis univariado. $1^{\text {ro }}$ de enero de 2015 hasta 30 de marzo de 2017

\begin{tabular}{|c|c|c|c|c|c|}
\hline Variables & $\begin{array}{c}\text { Con complicaciones } \\
\text { anestésicas } \\
\text { No }(\%) \\
\text { N }=47(21,17)\end{array}$ & $\begin{array}{c}\text { Sin complicaciones } \\
\text { anestésicas } \\
\text { No }(\%) \\
\mathrm{N}=\mathbf{1 7 5}(\mathbf{7 8 , 8 3 )}\end{array}$ & $\begin{array}{l}\text { Riesgo relativo } \\
\text { (RR) }\end{array}$ & $\begin{array}{l}\text { Intervalo de } \\
\text { confianza } \\
\text { (IC 95\%) }\end{array}$ & $p$ \\
\hline $\begin{array}{l}\text { Edad } \\
\geq 55 \text { años } \\
<55 \text { años }\end{array}$ & $\begin{array}{c}32(14,41) \\
15(6,76)\end{array}$ & $\begin{array}{c}101(45,50) \\
74(33,33)\end{array}$ & 1,56 & $0,84-2,88$ & 0,1965 \\
\hline $\begin{array}{l}\text { Tabaquismo } \\
\text { Si } \\
\text { No }\end{array}$ & $\begin{array}{c}27(12,16) \\
20(9,01)\end{array}$ & $\begin{array}{c}51(22,97) \\
124(55,86)\end{array}$ & 3,28 & $1,84-5,85$ & 0,0000 \\
\hline $\begin{array}{l}\text { Uso de beta-bloqueadores } \\
\text { No } \\
\text { Si }\end{array}$ & $\begin{array}{c}30(13,51) \\
17(7,66)\end{array}$ & $\begin{array}{c}74(33,33) \\
101(45,50)\end{array}$ & 2,40 & $1,32-4,86$ & 0,0045 \\
\hline $\begin{array}{l}\text { Uso de estatinas } \\
\text { No } \\
\text { Si }\end{array}$ & $\begin{array}{c}28(12,61) \\
19(8,56)\end{array}$ & $\begin{array}{c}69(31,08) \\
106(47,75)\end{array}$ & 2,26 & $1,26-4,05$ & 0,0074 \\
\hline
\end{tabular}

Fuente: Primaria.

Tabla 2. Factores de riesgo quirúrgico dependiente de enfermedades asociadas. Hospital Carlos Manuel de Céspedes. Análisis univariado. $1^{\text {ro }}$ de enero de $\mathbf{2 0 1 5}$ hasta 30 de marzo de 2017

\begin{tabular}{|c|c|c|c|c|c|}
\hline Variables & $\begin{array}{c}\text { Con complicaciones } \\
\text { anestésicas } \\
\text { No }(\%) \\
\mathrm{N}=47(21,17)\end{array}$ & $\begin{array}{c}\text { Sin complicaciones } \\
\text { anestésicas } \\
\text { No }(\%) \\
\mathrm{N}=\mathbf{1 7 5}(\mathbf{7 8 , 8 3 )}\end{array}$ & $\begin{array}{c}\text { Riesgo relativo } \\
\text { (RR) }\end{array}$ & $\begin{array}{l}\text { Intervalo de } \\
\text { confianza } \\
\text { (IC 95\%) }\end{array}$ & $p$ \\
\hline $\begin{array}{l}\text { Diabetes mellitus } \\
\text { Si } \\
\text { No }\end{array}$ & $\begin{array}{c}26(11,71) \\
21(9,46)\end{array}$ & $\begin{array}{c}49(22,07) \\
126(56,76)\end{array}$ & 3,18 & $1,79-5,65$ & 0,0001 \\
\hline $\begin{array}{l}\text { Arritmias } \\
\text { Si } \\
\text { No }\end{array}$ & $\begin{array}{c}19(8,56) \\
28(12,61)\end{array}$ & $\begin{array}{c}47(21,17) \\
128(57,66)\end{array}$ & 1,84 & $1,03-3,30$ & 0,0531 \\
\hline $\begin{array}{l}\text { Insuficiencia cardíaca } \\
\text { Si } \\
\text { No }\end{array}$ & $\begin{array}{l}27(12,16) \\
20(9,01)\end{array}$ & $\begin{array}{c}67(30,18) \\
108(48,65)\end{array}$ & 2,17 & $1,22-3,87$ & 0,0107 \\
\hline
\end{tabular}

Fuente: Primaria.

te de enfermedades asociadas y el pronóstico de aparición de complicaciones anestésicas se muestra en la Tabla 2. Se constata que presentar diabetes mellitus en la muestra de estudio se constituyó en un factor pronóstico al obtener RR superior a 1 (RR 3,18; IC 95\%: 1,79-5,65; $p=0,0001$ ).

De igual forma la insuficiencia cardíaca incrementó en dos el riesgo de aparición de complicaciones anestésicas en los pacientes con cardiopatía isquémica (RR 2,17; IC 95\%: 1,22-3,87; $\mathrm{p}=0,0107$ )

Mientras que la presencia de arritmias incrementó ligeramente el riesgo de complicaciones anestésicas (RR 1,84; IC 95\%: 1,03-3,30; $p=0,0531$ ).

La clasificación ASA III-IV en la muestra de estudio se constituyó en un factor pronóstico de desarrollar complicaciones anestésicas al obtener RR superior a 1 (RR 2,84; IC 95\%: 1,605,03; $p=0,0004)$.

En relación a los procedimientos quirúrgicos de alto riesgo triplicó el riesgo de desarrollar complicaciones anestésicas al obtenerse un valor del RR significativamente mayor de 1 (RR
3,58; IC 95\%: 1,83-6,98; p = 0,0001), constituyéndose así en la investigación en un factor pronóstico (Tabla 3).

En el análisis multivariado (Tabla 4) todas las variables objeto de estudio resultaron factores de riesgo para el pronóstico de aparición de complicaciones anestésicas. Fueron significativas estadísticamente: el no uso de estatinas con $R R=5,511$; intervalo de confianza de $(1,315$ : 23,104$)$ y $p=0,02$; el tabaquismo con $R R=19,993, p=0$ e intervalo desde 4,577 a 87,336; el procedimiento quirúrgico de alto riesgo con $R R=34,277, p=0$ e intervalo de confianza de 7,827 a 149,671; la diabetes mellitus con $R R=14,102$; intervalo de confianza de $(2,157 ; 92,221)$ y $p=0006$; la clasificación ASA con $R R=13,831, p=0$ e intervalo de confianza $(4,767 ; 40,127)$; el no uso de $\beta$-bloqueadores con $R R=6,566, p=0$ e intervalo de confianza $(2,158 ; 19,979)$ y la insuficiencia cardíaca con $R R=5,834$. No tuvieron significación estadística: la presencia de arritmias ( $R R=1,942 ; p=$ 0,285 e intervalo de confianza desde 0,576 a 6,549) y la edad mayor o igual de 55 años ( $R R=1,408 ; p=0,563$ e intervalo de confianza desde 0,442 a 4,490). 
Tabla 3. Factores de riesgo quirúrgico en función de la cirugía. Hospital Carlos Manuel de Céspedes. Análisis univariado. $1^{\text {ro }}$ de enero de 2015 hasta 30 de marzo de 2017

\begin{tabular}{|c|c|c|c|c|c|}
\hline Variables & $\begin{array}{c}\text { Con complicaciones } \\
\text { anestésicas } \\
\text { No }(\%) \\
\text { N }=47(21,17)\end{array}$ & $\begin{array}{c}\text { Sin complicaciones } \\
\text { anestésicas } \\
\text { No }(\%) \\
N=175(78,83)\end{array}$ & $\begin{array}{l}\text { Riesgo relativo } \\
\text { (RR) }\end{array}$ & $\begin{array}{l}\text { Intervalo de } \\
\text { confianza } \\
\text { (IC 95\%) }\end{array}$ & $p$ \\
\hline $\begin{array}{l}\text { Clasificación ASA } \\
\text { III-IV } \\
\text { II }\end{array}$ & $\begin{array}{l}25(11,26) \\
22(9,91)\end{array}$ & $\begin{array}{c}50(22,52) \\
125(56,31)\end{array}$ & 2,84 & $1,60-5,03$ & 0,0004 \\
\hline $\begin{array}{l}\text { Procedimientos quirúrgicos } \\
\text { de alto riesgo } \\
\text { Si } \\
\text { No }\end{array}$ & $\begin{array}{c}28(12,61) \\
19(8,56)\end{array}$ & $\begin{array}{c}51(22,97) \\
124(55,86)\end{array}$ & 3,58 & $1,83-6,98$ & 0,0001 \\
\hline
\end{tabular}

Fuente: Primaria.

Tabla 4. Factores de riesgo asociados a la aparición de complicaciones anestésicas en pacientes con cardiopatía isquémica sometidos a cirugía electiva con anestesia total intravenosa. Análisis multivariado. $1^{\text {ro }}$ de enero de 2015 hasta 30 de marzo de 2017. Nivel de significación 0,05

\begin{tabular}{|c|c|c|c|c|c|c|c|}
\hline \multirow[t]{2}{*}{ Variables } & \multirow[t]{2}{*}{ B } & \multirow{2}{*}{$\begin{array}{c}\text { Error } \\
\text { estándar }\end{array}$} & \multirow[t]{2}{*}{ Wald } & \multirow[t]{2}{*}{$p$} & \multirow[t]{2}{*}{$\mathbf{R R}$} & \multicolumn{2}{|c|}{$95 \%$ Intervalo de confianza } \\
\hline & & & & & & Inferior & Superior \\
\hline Arritmias & 0,664 & 0,620 & 1,144 & 0,285 & 1,942 & 0,576 & 6,549 \\
\hline Edad $\geq 55$ años & 0,342 & 0,592 & 0,335 & 0,563 & 1,408 & 0,442 & 4,490 \\
\hline Estatinas & 1,707 & 0,731 & 5,448 & 0,020 & 5,511 & 1,315 & 23,104 \\
\hline Tabaquismo & 2,995 & 0,752 & 15,855 & 0,000 & 19,993 & 4,577 & 87,336 \\
\hline Procedimiento quirúrgico & 3,533 & 0,753 & 22,027 & 0,000 & 34,227 & 7,827 & 149,671 \\
\hline Diabetes mellitus & 2,646 & 0,958 & 7,629 & 0,006 & 14,102 & 2,157 & 92,221 \\
\hline ASA & 2,627 & 0,543 & 23,365 & 0,000 & 13,831 & 4,767 & 40,127 \\
\hline$\beta$-bloqueadores & 1,882 & 0,568 & 10,988 & 0,001 & 6,566 & 2,158 & 19,979 \\
\hline Insuficiencia cardíaca & 3,353 & 0,532 & 9,855 & 0,000 & 5,834 & 0,646 & 5,206 \\
\hline Constante & $-10,862$ & 1,693 & 41,183 & 0,000 & 0,000 & & \\
\hline
\end{tabular}

\section{Conclusiones}

- El consumo de tabaco como hábito tóxico, el no uso de beta-bloqueadores y estatinas se constituyeron en los factores de riesgo quirúrgico en función del enfermo, asociados con el pronóstico de aparición de complicaciones anestésicas; no así la edad.

- La comorbilidad en los pacientes con cardiopatía isquémica de diabetes mellitus, insuficiencia cardíaca y las arritmias, se asociaron a la aparición de complicaciones anestésicas.

- La clasificación ASA III-IV y los procedimientos quirúrgicos de alto riesgo fueron los factores de riesgo quirúrgico en función de la cirugía, relacionados con el pronóstico de aparición de complicaciones anestésicas.

\section{Referencias}

1. Urrea JK, Yela Muñoz IE, Cifuentes C. Valoración perioperatoria del paciente para cirugía no cardíaca. Rev Colomb Cardiol. 2015;22(5):235-43. https://doi.org/10.1016/j.rccar.2015.02.007

2. Devereaux PJ, Xavier D, Pogue J, Guyatt G, Sigamani A, Garutti I, et al.; POISE (PeriOperative ISchemic Evaluation) Investiga- tors. Characteristics and short-term prognosis of perioperative myocardial infarction in patients undergoing noncardiac surgery: a cohort study. Ann Intern Med. 2011 Apr;154(8):523-8. https://doi.org/10.7326/0003-4819-154-8-201104190-00003 PMID:21502650

3. Lee TH, Marcantonio ER, Mangione CM, Thomas EJ, Polanczyk CA, Cook EF, et al. Derivation and prospective validation of a simple index for prediction of cardiac risk of major noncardiac surgery. Circulation. 1999 Sep;100(10):1043-9. https://doi. org/10.1161/01.CIR.100.10.1043 PMID:10477528

4. Fleisher LA, Fleischmann KE, Auerbach AD, Barnason SA, Beckman JA, Bozkurt B, et al. 2014 ACC/AHA guideline on perioperative cardiovascular evaluation and management of patients undergoing noncardiac surgery: a report of the American College of Cardiology/American Heart Association Task Force on Practice Guidelines. Circulation. 2014 Dec;130(24):e278-333. PMID:25085961

5. Kristensen SD, Knuuti J, Saraste A, Anker S, Bøtker HE, Hert SD, et al.; Authors/Task Force Members. 2014 ESC/ESA Guidelines on non-cardiac surgery: cardiovascular assessment and management: The Joint Task Force on non-cardiac surgery: cardiovascular assessment and management of the European Society of Cardiology (ESC) and the European Society of Anaesthesiology (ESA). 
Eur Heart J. 2014 Sep;35(35):2383-431. https://doi.org/10.1093/ eurheartj/ehu282 PMID:25086026

6. Dávila E. Anestesiología Clínica. Segunda Edición. La Habana: Editorial Ciencias Médicas; 2014.

7. Alcalá López JE, Maicas Bellido C, Hernández Simón P, Rodríguez Padial L. Cardiopatía isquémica: concepto, clasificación, epidemiología, factores de riesgo, pronóstico y prevención. Medicine (Baltimore). 2017;12(36):2145-52.

8. Fonnes S, Gögenur I, Søndergaard ES, Siersma VD, Jorgensen LN, Wetterslev J, et al. Perioperative hyperoxia - Long-term impact on cardiovascular complications after abdominal surgery, a post hoc analysis of the PROXI trial. Int J Cardiol. 2016 Jul;215:238-43. https://doi.org/10.1016/j.ijcard.2016.04.104 PMID:27128538

9. Myles PS, Leslie K, Chan MT, Forbes A, Peyton PJ, Paech MJ, et al.; ANZCA Trials Group for the ENIGMA-II investigators. The safety of addition of nitrous oxide to general anaesthesia in atrisk patients having major non-cardiac surgery (ENIGMA-II): a randomised, single-blind trial. Lancet. 2014 Oct;384(9952):144654. https://doi.org/10.1016/S0140-6736(14)60893-X PMID:25142708

10. Botto F, Alonso-Coello P, Chan MT, Villar JC, Xavier D, Srinathan $S$, et al.; Vascular events In noncardiac Surgery patlents cOhort evaluatioN VISION Study Investigators. Myocardial injury after noncardiac surgery: a large, international, prospective cohort study establishing diagnostic criteria, characteristics, predictors, and 30-day outcomes. Anesthesiology. 2014 Mar;120(3):56478. https://doi.org/10.1097/ALN.0000000000000113 PMID:24534856

11. Ramírez-Segura EH y col. Anestesia total intravenosa. Revista Mexicana de Anestesiología. 2015 Oct-Dec;38 Supl. 3:5430-2.

12. Obón Arellano Alfonso. Manejo actual del síndrome coronario agudo, primera parte: infarto del miocardio con onda Q [Internet]. Rev Costarric Cardiol. 2000 Dec;2(3):44-67. [cited 2019 Mar 5] Available from: http:// www.scielo.sa.cr/scielo.php?script=sci_arttext\&pid=S1409$41422000000300008 \&$ lng $=$ en

13. Trejo-Nava Carlos Alberto. Consideraciones para el manejo de la enfermedad arterial coronaria de adultos mayores. Rev. Mex. Cardiol [revista en la Internet]. 2014 Jun [citado 2019 Mar 15]; 25 (2) : 86-108. Disponible en: http://www.scielo.org.mx/scielo. php?script=sci_arttext\&pid=S0188-21982014000200004\&lng=es

14. Cohn SL. Updated guidelines on cardiovascular evaluation before noncardiac surgery: a view from the trenches. Cleve Clin J Med. 2014 Dec;81(12):742-51. https://doi.org/10.3949/ ccjm.81a.14148 PMID:25452352

15. Morales F. Abdomen agudo en el adulto mayor. Tratado de Geriatría y Gerontologia. San José, Costa Rica: EDNASSS-CCSS; 2015.

16. Wang $Y$, Zhao H, Zhou Z, Tian C, Xiao HL, Wang BE. Establishment and Application of Early Risk Stratification Method for Acute Abdominal Pain in Adults. Chin Med J (Engl). 2017 Mar;130(5):530-5. https://doi.org/10.4103/0366-6999.200553 PMID:28229983

17. Castellanos-Olivares A y cols. Prevalencia de comorbilidades en el paciente geriátrico y pronóstico postoperatorio. Revista Mexicana de Anestesiología. 2017;40(1):S103-5.

18. Fernández GE, Figueroa OD. Tabaquismo y su relación con las enfermedades cardiovasculares. Rev haban cienc méd [Internet]. 2018 Abr [citado 2019 Mar 16]; 17(2): 225-235. Disponible en: http://scielo.sld.cu/scielo.php?script=sci_arttext\&pid=S1729-

\section{$519 \times 2018000200008 \& \operatorname{lng}=\mathrm{es}$}

19. Vilches Izquierdo E, Ochoa Montes LA, Pernas Sánchez Y, González Lugo M, Ramos Marrero L, Tamayo Vicente ND, et al. Tabaquismo, consumo de alcohol y de café en el espectro de los factores de riesgo para la muerte cardíaca súbita. Rev cubana med [Internet]. 2014 Sep [citado 6 de marzo de 2018]; 53(3): 325-336. Disponible en: http://scielo.sld.cu/scielo.php?script=sci_ arttext\&pid=S0034-75232014000300009\&Ing=es

20. Achiong-Alemañy $M$, Achiong-Estupiñán $F$, Achiong-Alemañy $F$, Alfonso-de-León J, Álvarez-Escobar M, Suárez-Merino M. Riesgo cardiovascular global y edad vascular: herramientas claves en la prevención de enfermedades cardiovasculares. Rev Méd Electr [Internet]. 2016 [citado 6 de marzo de 2018]; 38(2):15. Disponible en: http://www.revmedicaelectronica.sld.cu/index.php/rme/ article/view/1641

21. Feng A. Peña Yamilé, Li Wan. La cardiopatía isquémica en pacientes diabéticos y no diabéticos. Rev haban cienc méd [Internet]. 2017 Abr [citado 2019 Mar 15]; 16(2): 217-228. Disponible en: http://scielo.sld.cu/scielo.php?script=sci_arttext\&pid=S1729$519 \times 2017000200008 \& \operatorname{lng}=\mathrm{es}$

22. Sionis A, Ruiz NJ, Fernández OA, Marín F, Abu AE, Díaz CO, et al. Actualización en cardiopatía isquémica y cuidados críticos cardiológicos. Revista Española de Cardiología. 2015;68(3):234-41. Consultado: 2016 Nov 3. Disponible en: http://www.revespcardiol.org/es/actualizacion-cardiopatia-isquemica-cuidados-criticos/ articulo/S0300893214006642/ https://doi.org/10.1016/j.recesp.2014.11.013.

23. Rodríguez NC. Evaluación del metabolismo hidrocarbonado y riesgo de diabetes en sujetos hospitalizados por cardiopatía isquémica. Málaga: Universidad de Málaga; 2014. Consultado: 2016 Nov 8. Disponible en: https:// oatd.org/oatd/record?record=oai\%5C\%3Adialnet.unirioja. es\%5C\%3ATES0000010775

24. Vázquez AC, Vaillant IM, González EJ. Comportamiento de la hipertensión arterial en pacientes con Diabetes mellitus tipo 2. Panorama Cuba y Salud. 2015;6(4):17-20. Consultado: 2016 Oct 29. Disponible en: http://wwwrevpanorama.sld.cu/index.php/ panorama/article/view/334

25. Higgins L. Clasificación del Estado Físico de la American Society of Anesthesiologists (ASA). Anestesiología Mexicana en Internet. Disponible en: http://www.anestesia.com.mx/asa.html (Último acceso 14 Nov 2013).

26. Qaseem A, Snow V, Fitterman N, Hornbake ER, Lawrence VA, Smetana GW, et al.; Clinical Efficacy Assessment Subcommittee of the American College of Physicians. Risk assessment for and strategies to reduce perioperative pulmonary complications for patients undergoing noncardiothoracic surgery: a guideline from the American College of Physicians. Ann Intern Med. 2006 Apr;144(8):575-80. https://doi.org/10.7326/0003-4819-144-8200604180-00008 PMID:16618955

27. Blum JM, Stentz MJ, Dechert R, Jewell E, Engoren M, Rosenberg $\mathrm{AL}$, et al. Preoperative and intraoperative predictors of postoperative acute respiratory distress syndrome in a general surgical population. Anesthesiology. 2013 Jan;118(1):19-29. https://doi. org/10.1097/ALN.0b013e3182794975 PMID:23221870

28. Brueckmann B, Villa-Uribe JL, Bateman BT, Grosse-Sundrup $M$, Hess $D R$, Schlett $C L$, et al. Development and validation of a score for prediction of postoperative respiratory complications. Anesthesiology. 2013 Jun;118(6):1276-85. https://doi. org/10.1097/ALN.0b013e318293065c PMID:23571640 